\title{
Diferencias del clima laboral entre empleados contratados por outsourcing y empleados directos: evidencia empírica de la zona norte de Colombia
}

Differences in the Working Climate of Employees Hired by Outsourcing and Employees Hired Directly: Empirical Evidence of the Northern Zone in Colombia

Diferenças do clima laboral entre empregados contratados por outsourcing e empregados diretos: evidência empírica da zona norte da Colômbia

\author{
David Juliao Esparragoza ${ }^{*}$ \\ Ciro Sánchez Bejarano* \\ Domingo Martínez Díaz
}

Fecha de recibido: 10 de mayo de 2013

Fecha de aprobado: 16 de diciembre de 2013

Doi: dx.doi.org/10.12804/rev.univ.empresa.26.2014.11

Para citar este artículo: Juliao E., D., \& Sánchez B., C., \& Martínez D., D. (2014). Diferencias del clima laboral entre empleados contratados por outsourcing y empleados directos: evidencia empírica de la zona norte de Colombia. Universidad \& Empresa, 16(26), 28 1-299. doi: dx.doi.org/10.12804/rev.univ.empresa.26.2014.11

\footnotetext{
Magíster en Administración de Empresas y Especialista en Mercadeo de la Universidad del Norte, Economista de la Universidad del Atlántico. Profesor de la Escuela de Negocios de la Universidad del Norte, Colombia. Correo electrónico: djuliae@uninorte.edu.co

** Estudiante de la Maestría en Administración de Empresas de la Universidad del Norte. Correo electrónico: ciroa.sanchez@gmail.com

*** Candidato a Doctor en Psicología de la Universidad del Norte, Magíster en Administración de Empresas de la Universidad de Norte, Especialista en mercados de la Universidad de los Andes, Estadístico de la Universidad de la Salle. Profesor investigador de la Escuela de Negocios de la Universidad del Norte, Colombia. Correo electrónico: domimart@uninorte.edu.co
} 


\section{RESUMEN}

Son pocos los estudios empíricos sobre clima laboral en Colombia que muestran las diferencias entre grupos de trabajadores que, teniendo las mismas funciones en la empresa, cuentan con diferentes tipos de contrato. Esta investigación, a partir de un estudio realizado con empleados de una importante empresa de bebidas ubicada en la costa norte de Colombia, trata de aportar a la resolución de esta carencia, verificando si existen diferencias significativas, en algunas variables del clima laboral, entre los grupos de trabajadores contratados directamente y aquellos vinculados por prestación de servicios. El trabajo es de tipo descriptivo y transversal, se apoya en el método hipotético inductivo y utiliza el instrumento IMCOC (evaluando las diferencias inter-grupales en las seis variables que lo componen, a saber: objetivos, cooperación, liderazgo, toma de decisiones, relaciones interpersonales, motivación y control).

Palabras clave: Clima laboral, IMCOC, outsourcing.

\section{ABSTRACT}

There are few empirical studies on working climate in Colombia that show differences among groups of workers who work under different types of labor contracts while having the same functions in the company. This research, based on a study of employees of an important beverage company located on the northern coast of Colombia intends to contribute to the solution of this gap by verifying whether there are significant differences between groups of workers hired directly and those hired under the mode of service providers (outsourcing). The study is descriptive and transversal; it relies on a hypothetical-inductive method and uses the IMCOC tool (that evaluates the differences among groups in terms of the six variables that integrate it, that is: objectives, corporations, leadership, decisionmaking, inter-personal relations, motivation and control).

Keywords: IMCOC, Outsourcing, Working environment.

\section{RESUMO}

São poucos os estudos empíricos sobre clima laboral na Colômbia que mostram as diferenças entre grupos de trabalhadores, que tendo a mesmas funções na empresa, contam com diferentes tipos de contrato. Esta pesquisa, a partir de um estudo realizado com empregados de uma importante empresa de bebidas localizada na Costa Norte da Colômbia, trata de aportar à resolução desta carência, verificando se existem diferenças significativas, em algumas variáveis do clima laboral, entre os grupos de trabalhadores contratados diretamente e aqueles vinculados por prestação de serviços. O trabalho é de tipo descritivo e transversal, apoia-se no método hipotético indutivo e utiliza o instrumento IMCOC (avaliando as diferenças intergrupais nas seis variáveis que o compõem, a saber: objetivos, cooperação, liderança, tomada de decisões, relações interpessoais, motivação e controle).

Palavras-chave: clima laboral, IMCOC, outsourcing. 


\section{INTRODUCCIÓN}

Para hacer medición del clima laboral existen diferentes opciones metodológicas. Es muy importante tener en cuenta, en cualquiera de ellas, las relaciones culturales como una parte fundamental de las organizaciones (Molero, 2002). Un punto muy relevante es diferenciar el clima laboral en la perspectiva de grupos (Robbins, 1996) ya que estos pueden tener sub-comportamientos que les son propios. Este artículo muestra los resultados de un trabajo empírico que, haciendo uso de la metodología de investigación científica, se propuso indagar respecto a la posible existencia de diferencias del clima laboral entre grupos con contratos directos $\mathrm{y}$ aquellos que trabajan para las empresas bajo la figura legal del outsourcing. Se parte de un cuestionario ampliamente reconocido como el $\mathrm{IMCOC}^{1}$ y se encuentra que estadísticamente la muestra revela comportamientos diferentes entre grupos, validando sus diferencias y afirmando que el clima laboral es mejor, en la mayoría de las varia- bles que compone el instrumento, cuando el empleado es contratado directamente por la empresa frente a la figura del outsourcing.

\section{REVISIÓN DE LA} LITERATURA

Existen múltiples aproximaciones al el concepto de "clima organizacional" (García, 2009). En la literatura se encuentran algunos ejercicios de asociación de conceptos sobre clima que permiten una clasificación organizada (Gómez, 2004). Así, las escuelas Funcionalista y de la Gestalt convergen en la necesidad que tienen los trabajadores de entender su entorno y adaptarse a él (Brunet, citado en Gómez, 2004).

Un trabajo que resume adecuadamente, de forma cronológica, las definiciones de diversos autores se encuentra en Álvarez (1992). La síntesis realizada por este autor se traduce en la Tabla 1. Ella permite ver las diferencias y convergencias que sobre el concepto tienen diversos autores representativos al interior de la literatura especializada.

1 El instrumento para medir clima en organizaciones colombianas (IMCOC) fue desarrollado por el profesor Carlos Eduardo Méndez Álvarez (2006) de la Universidad del Rosario y ha sido probado desde 1980 hasta 2005 en 176 empresas de diferentes sectores y tamaños en Colombia. 
Tabla 1. Aproximaciones sobre el concepto de clima organizacional

\begin{tabular}{|c|c|c|}
\hline Año & Autor & Definición \\
\hline 1939 & $\begin{array}{l}\text { Lewin, Lippit y } \\
\text { White }\end{array}$ & $\begin{array}{l}\text { El clima organizacional está relacionado de forma directa con las acti- } \\
\text { vidades de liderazgo. La reacción o clima de un grupo depende del tipo } \\
\text { de liderazgo al cual este es expuesto. La función de un líder es la de } \\
\text { crear una atmósfera que influya en el equipo. }\end{array}$ \\
\hline 1951 & Lewin & $\begin{array}{l}\text { El concepto de clima debe observarse en lo individual, es decir, hace } \\
\text { parte del ambiente sicológico (o ambiente de la persona). El clima tiene } \\
\text { como propósito unir los objetivos de la organización. }\end{array}$ \\
\hline 1955 & Francis Cornell & $\begin{array}{l}\text { Ingresa el término "percepciones" dentro de la acepción de clima, como } \\
\text { una combinación que permite entender la posición de las personas en } \\
\text { su trabajo. }\end{array}$ \\
\hline 1957 & Argyris & $\begin{array}{l}\text { Desarrolla el concepto teniendo en cuenta valores como confianza, } \\
\text { tranquilidad y otros que permiten dirimir problemas y resolverlos (to- } \\
\text { mado de Gómez, 2004). }\end{array}$ \\
\hline 1960 & Sells & $\begin{array}{l}\text { Está de acuerdo con Cornell en que la percepción del individuo influye } \\
\text { en su comportamiento. Involucra la aceptación o rechazo de las restric- } \\
\text { ciones sociales como condicionantes. }\end{array}$ \\
\hline 1963 & Halpin y Croft & Percepción que tiene un empleado sobre la organización. \\
\hline 1964 & Atkinson & $\begin{array}{l}\text { Introduce la "motivación promovida": es posible manipular o controlar } \\
\text { el clima a través de acciones empresariales. }\end{array}$ \\
\hline 1964 & $\begin{array}{l}\text { Forehand y } \\
\text { Gilmer }\end{array}$ & $\begin{array}{l}\text { Definen el clima como un conjunto de elementos claros e identificables } \\
\text { que expresan el carácter de una organización. Agregan la influencia que } \\
\text { este ejerce en el comportamiento de sus miembros. }\end{array}$ \\
\hline 1968 & $\begin{array}{l}\text { Littwin y Strin- } \\
\text { ger }\end{array}$ & $\begin{array}{l}\text { El clima es la visión compartida de los individuos relacionada con la } \\
\text { forma estructural de las organizaciones y el sistema de sanciones (to- } \\
\text { mado de Fernández, 2004). }\end{array}$ \\
\hline 1968 & Tagiuri y Litwin & $\begin{array}{l}\text { El clima puede ser descrito mediante un conjunto de características par- } \\
\text { ticulares de la organización. Es perdurable en el tiempo. }\end{array}$ \\
\hline 1968 & Pace & $\begin{array}{l}\text { Incluye el ambiente interno como parte esencial del clima, manifestan- } \\
\text { do que este es tomado como rasero para definir si existe o no percep- } \\
\text { ción de calidad. El resultado de este ejercicio es entendido como clima } \\
\text { laboral. Manifiesta también que este influye de forma directa a nivel } \\
\text { actitudinal entre sus miembros. }\end{array}$ \\
\hline 1971 & $\begin{array}{l}\text { Campbell, Dun- } \\
\text { nette, Lawler } \\
\text { y Weick }\end{array}$ & $\begin{array}{l}\text { El clima depende de la interacción de la empresa con su ambiente inter- } \\
\text { no y externo, mantienen la percepción como elemento crítico. }\end{array}$ \\
\hline 1972 & Hall & $\begin{array}{l}\text { El clima es una función del ambiente laboral que perciben de forma } \\
\text { directa o indirecta los trabajadores. Es influyente en la conducta del } \\
\text { empleado. }\end{array}$ \\
\hline
\end{tabular}




\begin{tabular}{|c|c|c|}
\hline Año & Autor & Definición \\
\hline $\begin{array}{l}1972 ; \\
1974\end{array}$ & $\begin{array}{l}\text { Pritchard y Kara- } \\
\text { sick; Helleriegel } \\
\text { y Slocum }\end{array}$ & $\begin{array}{l}\text { El clima se circunscribe como producto de la percepción, pero espe- } \\
\text { cifica su contexto inmediato. Se proponen once dimensiones para su } \\
\text { medición. }\end{array}$ \\
\hline 1974 & Dubrán & $\begin{array}{l}\text { El clima se puede traducir como la integración del ambiente interno de la } \\
\text { organización en términos de la sensación, la personalidad y el carácter. }\end{array}$ \\
\hline 1974 & James y Jones & $\begin{array}{l}\text { Hacen una diferencia importante entre el clima de la organización y el } \\
\text { clima psicológico. El primero atiende a las conductas, el segundo a las } \\
\text { motivaciones. }\end{array}$ \\
\hline 1976 & Cambell & $\begin{array}{l}\text { Incluye los procesos como generadores de comportamientos en la orga- } \\
\text { nización. Manifiesta que el clima está íntimamente relacionado con la } \\
\text { estructura de las operaciones. }\end{array}$ \\
\hline 1976 & Payne y Pugh & $\begin{array}{l}\text { Coinciden en que el clima se manifiesta a través de percepciones. Este } \\
\text { tiene en cuenta variables como actitudes, normas, sentimientos y con- } \\
\text { ductas. }\end{array}$ \\
\hline 1979 & Dessler & $\begin{array}{l}\text { Incluye los términos de estructura, recompensa y consideración como } \\
\text { parte esencial de la formación de opiniones como motor del clima. }\end{array}$ \\
\hline 1980 & $\begin{array}{l}\text { Naylor Pritchard } \\
\text { e Ilgen }\end{array}$ & $\begin{array}{l}\text { Establece al clima como cualidad duradera inmersa en el ambiente in- } \\
\text { terno de la organización. }\end{array}$ \\
\hline 1984 & Flippo & $\begin{array}{l}\text { Realiza un parangón entre el clima laboral y el meteorológico para con- } \\
\text { siderar cómo aquel es el resultado de múltiples variables que concurren } \\
\text { para crear un ambiente influyente en el comportamiento de los miembros. }\end{array}$ \\
\hline 1984 & Gibson et al. & $\begin{array}{l}\text { El clima es una mezcla de percepciones que involucra variables con- } \\
\text { ductuales y estructurales de la organización. Se incluye el concepto de } \\
\text { comunicación y las reacciones que sobre las acciones tomen los inte- } \\
\text { grantes de la organización. }\end{array}$ \\
\hline 1987 & Brunet & $\begin{array}{l}\text { Toma en cuenta no solo las variables relacionadas con la percepción y } \\
\text { el ambiente interno, en términos de satisfacción y productividad, sino } \\
\text { que incluye también el medio físico de la organización como variable } \\
\text { en la percepción del clima. }\end{array}$ \\
\hline 1990 & $\begin{array}{l}\text { Reichers } \\
\text { y Schneider }\end{array}$ & $\begin{array}{l}\text { El clima es una mezcla de percepciones que se comparten sobre los pro- } \\
\text { cedimientos en organizaciones formales o informales (Gómez, 2004). }\end{array}$ \\
\hline 1990 & Chiavenato & $\begin{array}{l}\text { Incluye como factores influyentes en el clima a la tecnología, las polí- } \\
\text { ticas, los reglamentos, los estilos de liderazgo, la etapa de la vida del } \\
\text { negocio, entre otros. Manifiesta que el clima genera dinámicas que pue- } \\
\text { den llevar a una mejor o menor productividad en la organización. }\end{array}$ \\
\hline 1990 & Robbins & $\begin{array}{l}\text { Explica el clima como la personalidad de la organización. Manifiesta } \\
\text { que es posible asimilarla bajo la cultura organizacional. }\end{array}$ \\
\hline 1992 & Álvarez & $\begin{array}{l}\text { Es la forma como el individuo expresa sus percepciones acerca del en- } \\
\text { torno del ambiente interno del cual participa en la organización. }\end{array}$ \\
\hline
\end{tabular}




\begin{tabular}{|c|c|c|}
\hline Año & Autor & Definición \\
\hline $\begin{array}{c}1992 \mathrm{a} \\
\mathrm{y} \\
1992 \mathrm{~b}\end{array}$ & Álvarez & $\begin{array}{l}\text { Manifiesta que el clima da paso a un sentido de pertenencia en la em- } \\
\text { presa que coadyuva a la satisfacción laboral. Se traduce en la manera de } \\
\text { un ambiente favorable o desfavorable en una organización. }\end{array}$ \\
\hline 1992 & $\begin{array}{l}\text { Gibson, } \\
\text { Ivancevich y } \\
\text { Donnelly }\end{array}$ & $\begin{array}{l}\text { Se hace un paralelo entre clima y cultura organizacional. Se indica que } \\
\text { los factores comunes que se manejan en estas son una pieza fundamen- } \\
\text { tal en la descripción del clima. }\end{array}$ \\
\hline 1995 & Peiró & $\begin{array}{l}\text { Hace énfasis en la subjetividad que marca el concepto. Establece el } \\
\text { clima como un equilibrio entre estructura y proceso. }\end{array}$ \\
\hline 1996 & Silva & $\begin{array}{l}\text { El clima se define a partir de las percepciones de la persona. Es una variable } \\
\text { integradora en los procesos que involucran a los grupos de una organización. }\end{array}$ \\
\hline 1997 & Goncalves & $\begin{array}{l}\text { El clima es una parte importante del proceso perceptual que manifiesta } \\
\text { tener un trabajador en relación con los procesos y estructuras en los que } \\
\text { están inmersos los medios laborales en los que él se encuentra. }\end{array}$ \\
\hline 2005 & Robles & $\begin{array}{l}\text { Involucra la dinámica de cambio que encierra el concepto a través del } \\
\text { tiempo como un fenómeno de percepciones sociales en la organización. }\end{array}$ \\
\hline 2003 & García & $\begin{array}{l}\text { El clima está compuesto de las percepciones y la opinión de un individuo } \\
\text { en relación con la organización, los factores de autonomía, estructura, } \\
\text { recompensas, consideración, cordialidad, apoyo, apertura, entre otros. }\end{array}$ \\
\hline 2006 & Méndez & $\begin{array}{l}\text { Es la dinámica cambiante que resulta de los procesos de interacción } \\
\text { social mediados por sistemas de valores, actitudes y creencias en el } \\
\text { ambiente interno de una organización. }\end{array}$ \\
\hline
\end{tabular}

Fuente: Elaboración propia, a partir de Álvarez (1992).

De acuerdo a lo anterior se puede ver una orientación de mayor estructura conforme se avanza en el tiempo ya que se incluyen conceptos más ricos en variantes y alcances en interactividad y relación de espacio. Se puede considerar como factor común el hecho de que la mayoría de autores define al constructo como el conjunto de percepciones que tienen sobre la organización los empleados de la misma (Alcantar, Maldonado, $\&$ Arcos, 2012).

Toro (1992), por su parte, ha hecho importantes aportes no solo al concepto sino a su difusión y aplicación en Colombia. En su libro El clima organizacional: perfil de empresas colombianas este autor (2001) visualiza el clima organizacional como indicador de la calidad de vida laboral. Del mismo modo, destaca la relación que tienen las variables demográficas en el comportamiento organizacional y su importancia en el desarrollo general de la empresa, todo en el ámbito colombiano.

Otros autores han realizado también aproximaciones al concepto en Co- 
lombia. Según Vega, Arévalo, Sandoval, Aguilar y Giraldo (2004) autores como Surdasky (1979) y Méndez (1982) han hecho importantes aportes y son motivadores iniciales del estudio del clima en Colombia.

Considerando la diversidad existente, y dada la precisión que este ofrece, se ha decido acercarse a una acepción del término "clima laboral" tomando como referente el trabajo de Stringer (2002). De acuerdo con este autor, el clima laboral es "la colección y el patrón de funcionamiento de los factores del entorno organizacional que generan motivación". En otras palabras, es la calidad del ambiente de trabajo que es experimentado por los miembros de una organización, el cual influye en su comportamiento.

Por otra parte, y dado que el ambiente interno varía de acuerdo con las condiciones laborales existentes, entre las que se encuentran los trabajos por prestación de servicios o las modalidades de outsourcing, se hace necesario revisar qué aproximaciones teóricas existen sobre la relación entre este tipo de contratación y los resultados de clima para personal que trabaja en las actividades de la organización.

Los contratos por prestación de servicios se muestran como una alter- nativa laboral que utilizan las empresas con el objetivo de adaptarse de manera eficiente a los ingentes cambios que produce la globalización(Vesga, 2011). La flexibilización laboral se muestra como una alternativa óptima para balancear el riesgo en la empresa, toda vez que permite concentrar recurso humano diversificado en áreas estratégicas y aquellas consideradas como más operativas. Esto, en términos porterianos, responde a aquellas que son fuente de ventajas competitivas y a las que no (Atkinson, 1984; Porter, 1997).

El clima laboral y el outsourcing han sido estudiados por algunos autores que manifiestan que este tipo de contratación tiene el objetivo de concentrar los esfuerzos empresariales en el núcleo o especialización de la compañía, dejando en manos de organizaciones subcontratistas (incluyendo a sus empleados) todas las labores que no se consideren indispensables o cuya inclusión no agrega valor substancial a la cadena (las revisiones de la cadena de valor porterianas pueden verse como adeptas a esta conducta). Sin embargo, este modelo tiene serios contradictores que aducen que la clase de contrato a seleccionar no está sujeta a una dirección estratégica basada en funciones, sino que está soportada en la disminución de los costos (Alles, 2000). 
Este amparo teórico, opuesto a la forma tradicional de ver el tipo de contrato inmerso en la cadena de valor porteriana, tiene profundas implicaciones pragmáticas que sería interesante estudiar para muchos trabajadores que se encuentran en el giro del negocio con diferentes tipos de contrato.

Lo cierto es que el enfoque que se le ha brindado a la relación subcontratación versus clima laboral no es fácil de encontrar, a pesar de la abundante literatura que existe sobre clima. Algunos trabajos se han acercado a la problemática tocando solo el aspecto de motivación como parte esencial del clima, manifestando, a través de un alcance empírico y de revisiones marginales de la literatura existente, una relación variada que no puede tomarse como rasero o ejemplo, ya que las condiciones ambientales pueden ser diferentes. A continuación se hace un abordaje de la relación que existe entre la subcontratación y la motivación de los trabajadores. Los descriptores de esta relación se encuentran en los trabajos de Bustamante (2010).

De acuerdo con este autor, a pesar de que existen teóricos que manifiestan que la relación de los sub- contratados con su motivación es buena, algunas narrativas muestran que esta solo es el producto de una esperanza que tienen los trabajadores subcontratados de pasar a la empresa madre. El trabajo evidencia que existen simbolismos culturales como pensar que la compañía subcontratista es buena, toda vez que les permite acercarse a la empresa madre. El estudio muestra que la esperanza de los trabajadores está en que la empresa subcontratista le permita al trabajador mostrarse y, si este es bueno, la empresa madre lo podría contratar.

\section{OBJETIVOS E HIPÓTESIS}

Como se dijo anteriormente, los contratos por prestación de servicios han sido considerados como una opción laboral válida que muchas empresas han implementado con la pretensión de adaptarse en forma eficiente a los grandes cambios generados gracias a la globalización. Por lo tanto, es inevitable que exista un alto grado de flexibilización laboral que lleve a la dirección de la organización a balancear el riesgo para la compañía, mediante la estrategia de concentrar el talento humano en las áreas estratégicas y operativas, tratando de aprovechar sus fuentes de ventaja competitiva. 
En este sentido, el objetivo de este trabajo es demostrar que el tipo de contratación laboral influye en el clima laboral.

Concretamente, la hipótesis plantea que el clima laboral de la empresa depende de la forma como se contrata la fuerza laboral. Ampliando esta hipótesis, se considera que existe un alto grado de dependencia entre el clima laboral respecto a si el trabajador es contratado directamente o si proviene de un contrato tipo outsourcing.

\section{METODOLOGÍA}

\section{A. Sujetos}

La muestra está formada por 389 empleados de una empresa de bebidas de la costa norte de Colombia que cuenta, en total, con 1.284 trabajadores. Están distribuidos en las ciudades de Barranquilla, Cartagena, Santa Marta, Sincelejo y Valledupar. De acuerdo con la ciudad de ubicación de los empleados, en la tabla 2 se aprecia la distribución de la muestra de los 389 sujetos.

Tabla 2. Distribución de la muestra según la ciudad

\begin{tabular}{l|c|c|c}
\hline \multicolumn{1}{c|}{ Ciudad } & Número de empleados & Porcentaje de empleados & Porcentaje válido \\
\hline Barranquilla & 149 & 38 & 38 \\
\hline Cartagena & 89 & 23 & 23 \\
\hline Sincelejo & 48 & 12 & 12 \\
\hline Santa Marta & 59 & 15 & 15 \\
\hline Valledupar & 44 & 12 & 12 \\
\hline Total & 389 & 100 & 100 \\
\hline
\end{tabular}

Fuente: Elaboración propia, usando el programa SPSS ${ }^{\circledR}$.

Para facilitar y viabilizar la hipótesis de trabajo, se consideró necesario clasificar a la población de estudio en tres subpoblaciones, denominadas arbitrariamente empresas 1, 2 y 3 , de la siguiente manera:

- Empresa tipo 1: Empresa ancla.

- Empresas tipo 2: Empresa que presta servicios de outsourcing a la empresa ancla.
- Empresas tipo 3: Empresa que presta servicios de outsourcing a la empresa ancla.

En la tabla 3 se relaciona la distribución de la muestra de 389 empleados, clasificada ahora según el tipo de empresa donde está vinculado el trabajador. 
Tabla 3. Distribución de la muestra según el tipo de empresa

\begin{tabular}{c|c|c|c|c}
\hline Empresa & $\begin{array}{c}\text { Número de empleados } \\
\text { en la población }\end{array}$ & $\begin{array}{c}\text { Número de empleados } \\
\text { en la muestra }\end{array}$ & Porcentaje & $\begin{array}{c}\text { Porcentaje } \\
\text { válido }\end{array}$ \\
\hline 1 & 49 & 16 & 4 & 4 \\
\hline 2 & 450 & 136 & 35 & 35 \\
\hline 3 & 785 & 237 & 61 & 61 \\
\hline Total & 1284 & 389 & 100 & 100 \\
\hline
\end{tabular}

Fuente: Elaboración propia, usando el programa SPSS ${ }^{\circledR}$.

Se utilizó un muestreo probabilístico estratificado, tomando como base de estratificación el tamaño de cada empresa. Para la determinación de los tamaños de muestra tanto de las subpoblaciones como de la población, se utilizó el procedimiento estadístico de Neyman, con un nivel de confianza del 95,44\% y un error muestral de seis meses, que corresponde a la variable meses trabajados. Los estratos según el tamaño y el tipo de empresa se definieron así:

- Empresa 1: Empresa con contrato laboral directo.

- Empresa 2: Empresa con contrato laboral indirecto.

- Empresa 3: Empresa con contrato laboral indirecto.

\section{B. Material y procedimiento}

Se partió del IMCOC, cuya validez empírica de los constructos definidos es conocida y aceptada. El IMCOC contiene las variables del comportamiento a través de la suma de preguntas relacionadas, es decir, las variables se consideran una relación lineal de las preguntas por las cuales se construyen. El cuestionario desarrolla el análisis de las siguientes variables sobre clima organizacional:

\section{Variable 1: Objetivos}

Esta variable se refiere al conocimiento que los trabajadores tienen sobre la razón de ser y los fines que persigue la empresa. Igualmente, ella evalúa la frecuencia con la que los trabajadores determinan las tareas que aseguran el cumplimiento de los objetivos de la empresa. Además, esta variable determina la calidad y periodicidad de la comunicación que los trabajadores reciben sobre estos aspectos.

\section{Variable 2: Cooperación}

La posibilidad de establecer procesos asociativos entre los miembros 
de la empresa en el desarrollo de sus labores permite el logro de los objetivos organizacionales. Esta variable busca medir actitudes y comportamientos específicos de colaboración de los trabajadores y el deseo de cooperar con los compañeros.

\section{Variable 3: Liderazgo}

Las preguntas de esta variable se refieren al ejercicio del liderazgo y la percepción que tienen los trabajadores de su jefe. En otras palabras, ellas apuntan a medir la relación que existe con el superior en la ejecución de los trabajos. Estas preguntas indagan también sobre la confianza que el jefe inspira.

\section{Variable 4: Toma de decisiones}

Esta variable se refiere a la posibilidad que tiene el trabajador de participar en el proceso de toma de decisiones, así como la imagen que refleja el jefe en el ejercicio de liderazgo. Entendemos la toma de decisiones como un subproceso de dirección que está relacionado con el estilo de liderazgo.

\section{Variable 5: Relaciones interpersonales}

Las preguntas formuladas en esta variable apuntan a conocer la frecuen- cia y forma como los trabajadores establecen relaciones interpersonales de carácter informal con sus compañeros de trabajo, así como el carácter positivo o negativo de las mismas.

\section{Variable 6: Motivación}

Esta variable se refiere a la relación que existe entre las actitudes de los trabajadores y la pertenencia a la organización. Ellas se expresan en niveles de satisfacción e indicadores de la motivación. Estas actitudes señalan comportamientos que llevan al trabajador al cumplimiento y eficacia de las funciones.

\section{Variable 7: Control}

Esta variable se refiere a la periodicidad y la forma como la empresa y los líderes ejercen el control sobre los trabajadores. A través del control la organización logra establecer si el trabajo realizado cumple o no con los objetivos de la misma.

\section{RESULTADOS Y ANÁLISIS}

Con los datos obtenidos se realizó inicialmente un resumen de las siete variables componentes del clima organizacional, según el instrumento, y cómo los diferentes tipos de empresas estudiadas se encuentran en cada una de estas, tal como se aprecia en la figura 1. 
Figura 1. Medias de las variables componentes del clima organizacional, por tipo de empresa, de acuerdo al IMcoC

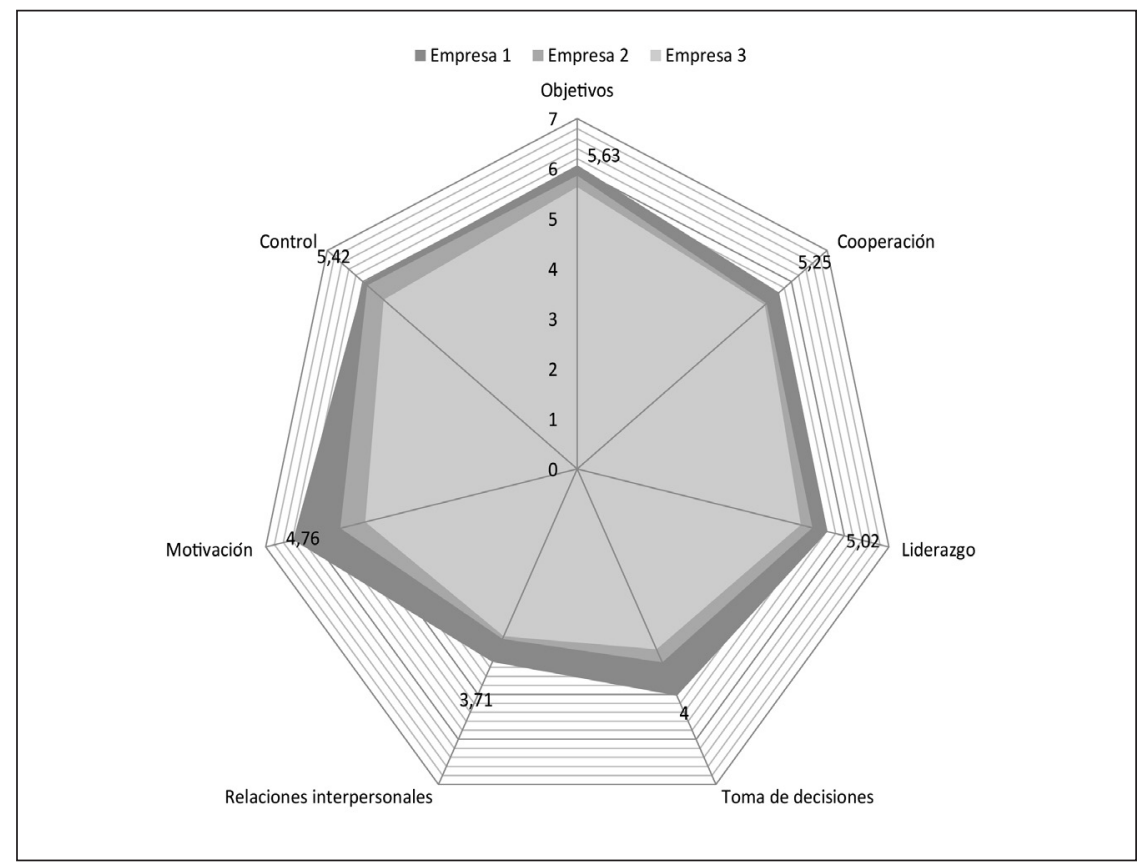

Fuente: Elaboración propia, a partir del IMCOC.

Se toma como punto de referencia que cualquier variable que esté mínimo con un promedio de cinco se considera que alcanza un buen comportamiento a favor del clima organizacional, de lo contrario, se recomienda que desarrolle acciones que mejoren el respectivo componente. Observando con atención la figura 1 , es posible verificar elementos en cada una de las empresas que son débiles en la construcción de un clima organizacional adecuado. La empresa 1 solo tiene en la variable relaciones interpersonales un puntaje inferior a cinco $(4,28)$, mostrando solidez en su comportamiento organizacional frente a las demás variables. A su vez, la empresa 2 muestra fallas en las relaciones interpersonales con un puntaje inferior a cinco $(3,77)$. Por último, la empresa 3 muestra en dos variables, relaciones interpersonales y toma de decisiones, indicadores inferiores a cinco (4 y 3,92, respectivamente). 
Tabla 4. Medias de las variables componentes del clima organizacional por tipo de empresa, de acuerdo a IMcoc

\begin{tabular}{c|c|c|c|c|c|c|c}
\hline Empresa & Objetivos & Cooperación & Liderazgo & $\begin{array}{c}\text { Toma de } \\
\text { decisiones }\end{array}$ & $\begin{array}{c}\text { Relaciones } \\
\text { interpersonales }\end{array}$ & Motivación & Control \\
\hline 1 & 6,0521 & 5,0417 & 5,8750 & 5,0125 & 4,4375 & 6,3375 & 6,0781 \\
\hline 2 & 5,8873 & 4,7377 & 5,4118 & 4,3897 & 3,6985 & 5,2588 & 5,6765 \\
\hline 3 & 5,6505 & 4,6308 & 5,1435 & 4,0810 & 3,7257 & 4,7907 & 5,1788 \\
\hline General & 5,7498 & 4,6851 & 5,2674 & 4,2272 & 3,7455 & 5,0180 & 5,3898 \\
\hline
\end{tabular}

Fuente: Elaboración propia, usando el programa SPSS ${ }^{\circledR}$.

En la tabla 4 se relacionan igualmente los promedios obtenidos en los siete componentes del clima organizacional, observándose que variables como relaciones interpersonales y toma de decisiones arrojaron valores críticos que deben mejorarse para todas las empresas, pero en particular para aquellas que manejan los contratos de tipo outsourcing. Los valores de estas componentes son más críticos en las empresas 2 y 3 . En estas variables se encontró que a los aspectos ambientales, que tienen que ver con la relación con compañeros, especialmente por fuera del horario laboral, se les debería prestar más atención. Asimismo, en el contexto empresarial estudiado las actividades de diversión que organiza la compañía no son percibidas como motivadoras. Esto conlleva una relación de clima, en el aspecto de relaciones interpersonales, más bajo. A ello se une la frecuencia con la cual los trabajadores toman deci- siones que solucion en problemas de trabajo. En este último, se descubrió que a medida que la relación de tamaño es mayor, existe también un mayor empoderamiento. Este hallazgo es interesante dado que se pretende demostrar que las poblaciones son diferentes.

La segunda parte del análisis corresponde a nuestra hipótesis de trabajo. La técnica estadística utilizada es el análisis de varianza, tomando como variable independiente el tipo de empresa que, como se dijo antes, tiene tres niveles:

Empresa 1: Empresa con contrato laboral directo.

Empresa 2: Empresa con contrato laboral indirecto.

Empresa 3: Empresa con contrato laboral indirecto.

Como variable dependiente -0 respuesta - se tomaron las evaluaciones dadas por lo empleados 
en las variables de clima organizacional.

\section{A. Planteamiento de las hipótesis}

Hipótesis nula:

$\mathrm{H}_{0}$ : No existe relación entre el tipo de contrato laboral (empresas) y las evaluaciones dadas por los empleados en las componentes del clima organizacional.
Hipótesis de trabajo:

$\mathrm{H}_{1}$ : El tipo de contrato laboral (empresas) influye significativamente en las evaluaciones dadas por los empleados en las componentes del clima organizacional.

Nivel de significancia: 5\%:

En la tabla 5 se plantean las hipótesis de manera estadística, lo que significa lo mismo que se plantea en su forma más general.

Tabla 5. Prueba de hipótesis para análisis de varianza

\begin{tabular}{l|l|l}
\hline \multicolumn{1}{c|}{$\begin{array}{c}\text { Componentes del clima } \\
\text { organizacional }\end{array}$} & Hipótesis nula & Hipótesis de trabajo \\
\hline Objetivos & $\mathrm{H}_{0}: \mu_{1}=\mu_{2}=\mu_{3}$ & $\mathrm{H}_{1}: \mu_{1} \neq \mu_{2} \neq \mu_{3}$ \\
\hline Cooperación & $\mathrm{H}_{0}: \mu_{1}=\mu_{2}=\mu_{3}$ & $\mathrm{H}_{1}: \mu_{1} \neq \mu_{2} \neq \mu_{3}$ \\
\hline Liderazgo & $\mathrm{H}_{0}: \mu_{1}=\mu_{2}=\mu_{3}$ & $\mathrm{H}_{1}: \mu_{1} \neq \mu_{2} \neq \mu_{3}$ \\
\hline Toma de decisiones & $\mathrm{H}_{0}: \mu_{1}=\mu_{2}=\mu_{3}$ & $\mathrm{H}_{1}: \mu_{1} \neq \mu_{2} \neq \mu_{3}$ \\
\hline Relaciones interpersonales & $\mathrm{H}_{0}: \mu_{1}=\mu_{2}=\mu_{3}$ & $\mathrm{H}_{1}: \mu_{1} \neq \mu_{2} \neq \mu_{3}$ \\
\hline Motivación & $\mathrm{H}_{0}: \mu_{1}=\mu_{2}=\mu_{3}$ & $\mathrm{H}_{1}: \mu_{1} \neq \mu_{2} \neq \mu_{3}$ \\
\hline Control & $\mathrm{H}_{0}: \mu_{1}=\mu_{2}=\mu_{3}$ & $\mathrm{H}_{1}: \mu_{1} \neq \mu_{2} \neq \mu_{3}$ \\
\hline
\end{tabular}

Fuente: Elaboración propia.

Descrito lo anterior, se corren siete análisis de varianza (ANOVA), en el paquete estadístico SPSS ${ }^{\circledR}$, obteniéndose las diferencias en las medias poblacionales.

B. Resultados de los análisis de varianza

Tomando como nivel de significancia un valor del $5 \%$, para el rechazo de la hipótesis nula, podemos ver en la tabla 6 que cinco de las siete variables explicativas del clima organizacional, siguiendo el modelo de Méndez (2006), son altamente significativas. Esto conduce al rechazo de la hipótesis nula.

Esto significa además que, definitivamente, el tipo de contrato influye en el clima laboral. Además, el clima organizacional se va deteriorando a medida que se avanza de un 
contrato directo hacia un contrato del tipo outsourcing; en otras palabras, los empleados que gozan de una contratación directa perciben un mejor clima laboral, especialmente en aspectos que se relacionan con el liderazgo, la toma de decisiones, la motivación y el control. Sin embargo, componentes como objetivos, liderazgo y control, que gozan de niveles adecuados de clima laboral (mayores que cinco) en las tres empresas, mostraron igualmente diferencias significativas a favor de la empresa donde los empleados tienen contratación directa.

En las variables cooperación y relaciones interpersonales la prueba aceptó la hipótesis nula. Esto significa que el tipo de contrato no influye en el clima laboral (existe independencia estadística). Este resultado no demuestra la hipótesis de trabajo de los investigadores, pero, si se mira de otra forma, es en estos dos componentes en donde se presentó el peor clima organizacional. Sus promedios están por debajo del umbral. Esto invitaría a la empresa, en general, a desarrollar acciones para que los empleados puedan fortalecer estos dos componentes, pues son fundamentales para un mejor trabajo, es decir, uno apoyado, justamente, en una buena cooperación y en unas excelentes relaciones interpersonales.

Tabla 6. Prueba anova sobre variables de clima, de acuerdo al tipo de empresa

\begin{tabular}{|c|c|c|c|c|c|c|}
\hline $\begin{array}{c}\text { Componentes del } \\
\text { clima organizacional }\end{array}$ & Nivel & $\begin{array}{l}\text { Suma de } \\
\text { cuadrados }\end{array}$ & gl & $\begin{array}{c}\text { Media } \\
\text { cuadrática }\end{array}$ & $\mathrm{F}$ & Sig. \\
\hline \multirow{3}{*}{ Objetivos } & Inter-grupos & 6.369 & 2 & 3.184 & 4.166 & .016 \\
\hline & Intra-grupos & 295.055 & 386 & .764 & & \\
\hline & Total & 301.424 & 388 & & & \\
\hline \multirow{3}{*}{ Cooperación } & Inter-grupos & 3.110 & 2 & 1.555 & 1.582 & .207 \\
\hline & Intra-grupos & 379.397 & 386 & .983 & & \\
\hline & Total & 382.507 & 388 & & & \\
\hline \multirow{3}{*}{ Liderazgo } & Inter-grupos & 12.382 & 2 & 6.191 & 7.872 & .000 \\
\hline & Intra-grupos & 303.574 & 386 & .786 & & \\
\hline & Total & 315.955 & 388 & & & \\
\hline \multirow{3}{*}{ Toma de decisiones } & Inter-grupos & 18.524 & 2 & 9.262 & 9.282 & .000 \\
\hline & Intra-grupos & 385.148 & 386 & .998 & & \\
\hline & Total & 403.671 & 388 & & & \\
\hline
\end{tabular}




\begin{tabular}{l|l|c|c|c|c|c}
\hline $\begin{array}{c}\text { Componentes del } \\
\text { clima organizacional }\end{array}$ & \multicolumn{1}{|c|}{ Nivel } & $\begin{array}{c}\text { Suma de } \\
\text { cuadrados }\end{array}$ & gl & $\begin{array}{c}\text { Media } \\
\text { cuadrática }\end{array}$ & F & Sig. \\
\hline \multirow{4}{*}{ Relaciones interpersonales } & Inter-grupos & 8.054 & 2 & 4.027 & 2.019 & .134 \\
\cline { 2 - 8 } & Intra-grupos & 769.750 & 386 & 1.994 & & \\
\cline { 2 - 8 } & Total & 777.805 & 388 & & & \\
\hline \multirow{4}{*}{ Motivación } & Inter-grupos & 47.988 & 2 & 23.994 & 25.036 & .000 \\
\cline { 2 - 8 } & Intra-grupos & 369.926 & 386 & .958 & & \\
\cline { 2 - 8 } Control & Total & 417.914 & 388 & & & \\
\hline & Inter-grupos & 29.309 & 2 & 14.654 & 14.790 & .000 \\
\cline { 2 - 8 } & Intra-grupos & 382.450 & 386 & .991 & & \\
\cline { 2 - 8 } & Total & 411.759 & 388 & & & \\
\hline
\end{tabular}

Fuente: Elaboración propia, usando el programa SPSS ${ }^{\circledR}$.

Esta prueba cuenta con el contraste de homogeneidad de varianzas (prueba de Levene), la cual confirma este supuesto como indicador de un resultado confiable (ver tabla 7).

\section{DISCUSIÓN Y \\ CONCLUSIONES}

Los resultados obtenidos pueden explicarse considerando la existencia de un componente teórico que muestra que las relaciones entre el tipo de contrato son influyentes en la percepción que tiene el trabajador sobre su medio. Este componente es estudiado por la teoría del contrato sicológico. Esta teoría manifiesta que el nivel de expectativas al cual se enfrenta cada trabajador es mediado por el tipo de vinculación laboral que tenga (Coyle-Shapiro \& Kessler 2000; Tena, 2002; Vesga, 2011).

Otro aspecto que ha sido estudiado es el significado que se construye acerca del trabajo mediante procesos cognitivos, sociales y políticos (Palaci, citado en Vesga 2011). De acuerdo con estos significados es posible comprender las creencias, atributos y mitos relacionados que forman parte de la experiencia construida socialmente.

Tabla 7. Prueba de homogeneidad de varianzas

\begin{tabular}{l|c|c|c|c}
\hline Componentes del clima organizacional & Estadístico de Levene & gl1 & g12 & Sig. \\
\hline Objetivos & 5.164 & 2 & 386 & .006 \\
\hline Cooperación & 2.838 & 2 & 386 & .060 \\
\hline Liderazgo & 3.970 & 2 & 386 & .020 \\
\hline
\end{tabular}




\begin{tabular}{l|c|c|c|c}
\hline \multicolumn{1}{c|}{ Componentes del clima organizacional } & Estadístico de Levene & $\mathrm{gl1}$ & $\mathrm{gl}$ & $\mathrm{Sig}$. \\
\hline Toma de decisiones & 1.982 & 2 & 386 & .139 \\
\hline Relaciones interpersonales & 3.932 & 2 & 386 & .020 \\
\hline Motivación & 4.620 & 2 & 386 & .010 \\
\hline Control & 6.021 & 2 & 386 & .003 \\
\hline
\end{tabular}

Fuente: Elaboración propia, usando el programa SPSS ${ }^{\circledast}$.

Al parecer, esta experiencia tiene factores comunes que son explicados por el tipo de contrato ya que los significados sociales y aceptados son, de hecho, comunes. Para el caso de los empleados en forma de contratación indirecta -outsourcing-, Vesga (2011) manifiesta que esta clase de vinculación tiene connotaciones negativas, toda vez que es percibida socialmente como de alto riesgo, vinculada con un menor salario y en cercanía a un carácter discriminante. Sin embargo, se encuentra que es posible que la relación laboral mantenga una productividad aceptada ya que esta relación en esta clase de trabajadores (contratos implícitos) está condicionada por la expectativa de que su desempeño pueda ser una plataforma para el cambio de contrato.

Es necesario destacar que las relaciones encontradas a través de la presente investigación revelan que el comportamiento organizacional es más sólido en las empresas de mayor tamaño. Por lo anterior, se podría presumir que es posible que sí exista en la práctica el mito de que estar más cerca de la empresa ancla es mejor. Este factor es explicado a través del fenómeno del contrato sicológico. Es importante que este contrato no se viole, es decir, que la expectativa del trabajador relacionada con el cambio de modalidad de empleo tiene un período de tensión "sana", el cual, una vez se rompe, hace que se corra el riesgo de tener rendimientos marginales decrecientes, pues el trabajador quiebra la expectativa y es posible que su desmotivación genere fallas en sus procesos habituales.

Se concluye que, a excepción de las variables de relaciones interpersonales y de cooperación, todas las demás variables del clima organizacional consideradas en el IMCOC se ven influenciadas por el tipo de contrato en la muestra estudiada. Si bien los resultados de la muestra no se pueden extrapolar, resulta de especial cuidado la revisión de las expectativas y cómo estas influyen en la dinámica del entorno y en la productividad del empleado ( $c f$. Seisdedos, 1985). 


\section{REFERENCIAS}

Alcántar, V., Maldonado, S., \& Arcos, J. L. (2012). Medición del clima laboral requerido para asegurar la efectividad del sistema de gestión de calidad. Revista internacional administración $y$ finanzas, 5(3), 55-68.

Alles, M. (2000). Dirección estratégica de recursos humanos. Buenos Aires: Granica.

Álvarez, G. (1992). El constructo "clima organizacional": concepto, teorías, investigaciones y resultados relevantes. Revista Interamericana de Psicología ocupacional, 11(1-2), 25-47.

Bermúdez R., H., \& Bustamante S., A. M. (2010). La motivación de los trabajadores subcontratados. Una paradoja incesante. Ad-Minister, 17, 35-57.

Brunet, L. (1999). El clima de trabajo en las organizaciones: definiciones, diagnóstico y consecuencias. México: Trillas.

Contreras, F., Juárez, F., Barbosa, D., \& Uribe, A. F. (2010). Estilos de liderazgo, riesgo psicosocial y clima organizacional en un grupo de empresas colombianas. Revista Facultad de Ciencias Económicas, Investigación y Reflexión, XVIII(2), 7-17.

Coyle-Shapiro, J., \& Kessler, I. (2000). Consequences of the psychological contract for the em- ployment relationship: A large scale survey. Journal of Management Studies, 37(7), 903-930.

García G., M. E., Escalante D., M., \& Quiroga S. A., Y. (2012). Importancia del clima laboral en los resultados de una empresa y la competitividad. Contribuciones a la Economía. Recuperado de http://www.eumed.net/ ce/2012/clima-laboral-empresa-competitividad.html

García, M. (2009). Clima organizacional y su diagnóstico: una aproximación conceptual. Cuadernos de Administración, 42, 43-61. Gómez Rada, C. (2004). Diseño, construcción y validación de un instrumento que evalúa clima organizacional en empresas colombianas, desde la teoría de respuesta al item. Bogotá: Universidad Católica de Colombia, Facultad de Psicología.

Mejía R., C., Contreras, F., Barbosa, D., Juárez, F., \& Uribe, A. F. (2009). Estilos de liderazgo, clima organizacional y riesgos psicosociales en entidades del sector salud como criterios de responsabilidad social empresarial. Estudio comparativo en cuatro ciudades colombianas. Acta Colombiana de Psicología, 12(2), 13-26.

Méndez Á., C. E. (2006). Clima organizacional en Colombia. El IMCOC: un método de análisis 
para su intervención. Bogotá: Centro Editorial Universidad del Rosario.

Molero, F. (2002). Cultura y liderazgo. Una relación multifacética. Boletín de Psicología, 76, 53-75. Robbins, S. P., \& Deras Q. A. (1996). Comportamiento organizacional: teoría y práctica México: Prentice Hall Hispanoamericana. Seisdedos, N. (1985). El clima laboral y su medida. Madrid: Colegio Oficial de Psicólogos de Madrid. Stringer, R. (2002). Leadership and organizational climate. Nueva Jersey: Prentice Hall.

Tena, G. (2002). El contrato psicológico: relación laboral empresatrabajador. Acciones e investigaciones sociales, 15, 85-107.
Toro, F. (1992). Desempeño y productividad. Contribuciones de la psicología organizacional. Medellín: Cincel.

Toro, G., Ochoa, L., \& Vargas, A. (2004). Clima organizacional: promotor de la creatividad en la empresa. Revista Creando, 2(3), 1-7.

Vega, D, Arévalo, A., Sandoval, J., Aguilar, M. C., \& Giraldo, J. (2004). Panorama sobre los estudios de clima organizacional en Bogotá, Colombia (1994-2005). Diversitas, 2(002), 329-349.

Vesga R., J. J. (2011). Los tipos de contratación laboral y sus implicaciones en el contrato psicológico. Pensamiento Psicológico, 9(16), 171-182. 